\title{
PROJECTIVE MODULI OF CERTAIN QUOTIENT RINGS
}

\author{
NADINE MOORE
}

\begin{abstract}
The author considers some properties of extension rings $B$ of a $\operatorname{ring} A$ that satisfy the condition that every maximal ideal of $B$ is an extension of some ideal of $A$. Such extensions have been used by $\mathrm{D}$. Lissner, $\mathrm{K}$. Lønsted, N. Moore, and A. Simis to obtain rings for which the projective moduli are arbitrarily less than the dimension of the maximal spectra. It is shown that families of prime ideals of maximal type can be used to construct such extensions.
\end{abstract}

Introduction. Throughout this paper all rings are assumed to be commutative with an identity and all extension rings are assumed to be unitary extensions. If $B$ is an extension ring of a $\operatorname{ring} A$, the pair $(B, A)$ is said to have Property $E_{M}$ if every maximal ideal of $B$ can be written as $I B$ for some ideal $I$ of $A$.

We now list some results concerning pairs of rings with Property $E_{M}$. The proofs are elementary and are omitted.

RESULT 1.1. If $B$ is an extension ring of a ring $A$, then a necessary and $a$ sufficient condition for $(B, A)$ to have Property $E_{M}$ is that $M=(M \cap A) B$ for each maximal ideal $M$ of $B$.

RESULT 1.2. Suppose $C$ is an extension ring of $B$ and $B$ is an extension ring of a ring $A$.

(a) If $(C, A)$ has Property $E_{M}$, then $(C, B)$ has Property $E_{M}$.

(b) If $C$ is an integral extension of $B$ and if both $(C, B)$ and $(B, A)$ have Property $E_{M}$, then $(C, A)$ has Property $E_{M}$.

Result 1.3. If $B$ is an extension ring of a ring $A$ and $(B, A)$ has Property $E_{M}$, then $[B / I, A /(I \cap A)]$ has Property $E_{M}$ for each ideal I of $B$.

For a ring $A, \max A$ denotes the maximal spectrum of $A$ with the Zariski topology [1, Chapter III, §3].

THeOREM 1.4. Suppose $B$ is an extension ring of $a$ ring $A$ and $(B, A)$ has Property $E_{M}$.

(a) If $m \in \max A$, then $m B=B$ or $m B \in \max B$.

(b) If $a B \neq B$ for all proper ideals $a$ of $A$, then the mapping $M \rightarrow M \cap A$ defines a continuous bijection of $\max B$ into $\max A$. The inverse mapping is given by $m \rightarrow m B$.

Received by the editors June 24, 1974.

AMS (MOS) subject classifications (1970). Primary 13B99; Secondary $13 \mathrm{C} 10$.

Key words and phrases. Projective modulus, families of prime ideals of maximal type, maximal spectrum, unimodular element, integral extension. 
Proof. Suppose $m \in \max A$ is such that $m B \neq B$. Then $m B \subseteq M$ for some maximal ideal $M$ of $B$; hence $m \subseteq M \cap A \neq A$. Therefore $m=M \cap A$ and $M=(M \cap A) B=m B$.

To prove condition (b), we let $f$ be the mapping from max $B$ defined by $f(M)=M \cap A$, and we let $g$ be the mapping from $\max A$ defined by $g(m)=m B$. Condition (a) implies $g: \max A \rightarrow \max B$. We now show that $f: \max B \rightarrow \max A$. Suppose $M \in \max B$. Since $M \cap A \neq A$, there is a maximal ideal $m$ of $A$ which contains $M \cap A$. Then $M=(M \cap A) B \subseteq m B$ $\neq B$; it follows that $M=m B$. Hence $M \cap A=m$. We have proved $M \cap A$ $\in \max A$ and $g f(M)=M$. If $m \in \max A$, then $f g(m)=m B \cap A=m$. It is well known that if $h: A \rightarrow B$ is a ring homomorphism, then the map $\operatorname{spec} B \rightarrow \operatorname{spec} A$ given by $p \rightarrow h^{-1}(p)$ is continuous. By letting $h$ be the inclusion mapping, we deduce that $f$ is continuous.

Proposition 1.5 [3], [6]. If $B$ is an integral extension of $A$ such that for each maximal ideal $m$ of $A$ there is a unique ideal of $B$ which lies over $m$, then $M \rightarrow M \cap A$ defines a homeomorphism from $\max B$ onto $\max A$.

2. Unimodular elements. Throughout this section $B$ is an extension ring of a ring $A$. If $X$ is a ring and $P$ is an $X$-module, an element $u$ of $P$ is called $X$ unimodular if $f(u)$ is a unit of $X$ for some $f \in \operatorname{Hom}_{X}(P, X)$. If $P$ is a $B$-module, we regard $P$ also as an $A$-module in the usual way.

Proposition 2.1 [3]. Suppose $X$ is a ring and $P$ is an $X$-module.

(a) If $u \in P$ is $X$-unimodular, then $u \notin m P$ for all $m \in \max X$.

(b) If $P$ is a projective $X$-module and $u \notin m P$ for all $m \in \max X$, then $u$ is $X$-unimodular.

Corollary 2.2. Suppose $(B, A)$ has Property $E_{M}$ and $a B \neq B$ for all proper ideals a of $A$. Suppose also that $P$ is a $B$-module which is projective as an $A$ module. If $u \in P$ is $B$-unimodular, then $u$ is $A$-unimodular.

Corollary 2.3. Suppose $(B, A)$ has Property $E_{M}$ and suppose $P$ is a projective $B$-module. If $u \in P$ is $A$-unimodular, then $u$ is also $B$-unimodular.

The projective modulus of a ring $X$, denoted $\mathrm{pm} X$, is the least nonnegative integer $k$ such that every projective $X$-module is the direct sum of a free module and a module of rank $\leqslant k$.

Corollary 2.4. Suppose $B$ is a projective $A$-module for which $0<f$ - $\operatorname{rank}_{A} B=d<\infty$ [see [1] for definition], $\max B$ is noetherian, and $(B, A)$ has Property $E_{M}$. Then $\mathrm{pm} B \leqslant \mathrm{pm} A / d$. If $B$ is also an integral extension of $A$, then $\mathrm{pm} B \leqslant \operatorname{dim} \max B / d$.

The proof is in [3].

The ring extension $B$ in Corollary 2.4 for which $f-\operatorname{rank}_{A} B=d>1$ satisfies the inequality that $\operatorname{dim} \max B>\mathrm{pm} B$. For such rings Corollary 2.4 is an improvement over Serre's theorem [1] that $\operatorname{dim} \max B \geqslant \mathrm{pm} B$.

3. Integral extensions. Throughout this section we assume $B$ is an integral extension of $A$. Let $\left\{P_{i}\right\}$ be a family of prime ideals of $A$ and $T=A-\cup P_{i}$. The family $\left\{P_{i}\right\}$ is said to be of maximal type [6] if every maximal ideal of $T^{-1} A$ 
is of the form $T^{-1} P_{i}$ for some $i$, or equivalently if any ideal of $A$ maximal among the ideals not intersecting $T$ is some $P_{i}$. If there are no inclusion relations among the $P_{i}$ 's then $\max T^{-1} A=\left\{T^{-1} P_{i}\right\}[6]$.

THEOREM 3.1. Suppose $\left\{P_{i}\right\}$ is a family of prime ideals of $A$ of maximal type such that there are no inclusion relations among the $P_{i}$ 's. Let $\left\{Q_{j}\right\}$ be the family of all prime ideals $Q$ of $B$ such that $Q \cap A=P_{i}$ for some $i$. Let $T=A-\cup P_{i}$ and $S=B-\cup Q_{i}$. Then:

(a) $T^{-1} B=S^{-1} B$;

(b) $\left\{Q_{j}\right\}$ is a family of prime ideals of $B$ of maximal type such that there are no inclusion relations among the $Q_{j}$ 's.

Proof. There are no inclusion relations among the $Q_{j}$ 's by the CohenSeidenberg going-up lemma. The major part of the proof consists of showing that $\max T^{-1} B=\left\{Q_{j}\left(T^{-1} B\right)\right\}$. For each $j, Q_{j} \cap T=\varnothing$ and $Q_{j}\left(T^{-1} B\right)$ is a prime ideal of $T^{-1} B$. The maximal ideals of $T^{-1} B$ are those ideals of the form $M\left(T^{-1} B\right)$ where $M$ is an ideal of $B$ which is maximal with respect to the property that $M \cap T=\varnothing$. Since $T^{-1} B$ is an integral extension of $T^{-1} A$, for such $M$ 's $(M \cap A) T^{-1} A=M\left(T^{-1} B\right) \cap T^{-1} A$ is a maximal ideal of $T^{-1} A$. It follows that $M \cap A=P_{i}$ for some $i$. Then $M=Q_{j}$ for some $j$. We have proved max $T^{-1} B \subseteq\left\{Q_{j}\left(T^{-1} B\right)\right\}$. Since any $Q_{j}\left(T^{-1} B\right)$ is contained in some maximal ideal of $T^{-1} B$ of the type $Q_{k}\left(T^{-1} B\right)$, it follows that $Q_{j} \subseteq Q_{k}$; hence $Q_{j}=Q_{k}$. To prove Condition (a) let $s \in S$. For all $j, s \notin Q_{j}$; hence $s \notin Q_{j}\left(T^{-1} B\right)$. Therefore $s$ is a unit of $T^{-1} B$. Condition (b) is a trivial consequence of Condition (a) and the preceding remarks.

CoRollary 3.2. Suppose $\left\{P_{i}\right\}$ is a family of prime ideals of $A$ of maximal type such that $P_{i} B=Q_{i}$ is a prime ideal of $B$ for each $i$. Let $S=B-\cup Q_{i}$ and $T=A-\cup P_{i}$. Then:

(a) $S^{-1} B=T^{-1} B$.

(b) $\left(S^{-1} B, T^{-1} A\right)$ has Property $E_{M}$.

(c) $\max S^{-1} B$ is homeomorphic to $\max T^{-1} A$.

(d) If $B$ is a projective $A$-module of $f-$ rank $d \quad(0<d<\infty)$ and $\max B$ is noetherian, then $\mathrm{pm} S^{-1} B \leqslant \operatorname{dim} \max S^{-1} B / d$.

Proof. It follows from the Cohen-Seidenberg going-up lemma that for each $i, Q_{i}$ is the unique prime ideal of $B$ which lies over $P_{i}$. The corollary now follows from Theorem 3.1, Proposition 1.5, and Corollary 2.4.

EXAMPLE. Suppose $F$ is a field which is not algebraically closed and $K$ is a finite extension field of $F . F[X]$ denotes the ring of polynomials $F\left[X_{1}, \ldots, X_{n}\right]$ in $n$ indeterminates; similarly for $K[X]$. Let $I$ be a proper ideal of $F[X]$ and $V$ be a nonempty set in the variety of $I$ in $F^{n}=F \times \cdots \times F$. Let $J=I K[X]$. Then [3] we have $J \cap F[X]=I$ and the map $A=F[X] / I \rightarrow K[X] / J=B$ is a monomorphism, which we regard as an inclusion. We consider $A$ as a ring of functions from $V$ into $F$ and $B$ as a ring of functions from $V$ into $K$. For each $v \in V$, let $M_{v}=\{f \in A: f(v)=0\}$ and $N_{v}=\{f \in B: f(v)=0\}$. $\left\{M_{v}: v \in V\right\}$ is a family of prime ideals of $A$ of maximal type [6]. $\left\{N_{v}: v \in V\right\}$ is a family of prime ideals of $B$. For each $v \in V, N_{v}=M_{v} B$ [3]. Let $T=A-\cup M_{v}=\{f \in A: f(v) \neq 0$ for all $v \in V\}$ and $S=B-\cup N_{v}$ 
$=\{f \in B: f(v) \neq 0$ for all $v \in V\}$. Corollary 3.2 applies in this case.

One example [3], [5] that the upper bound in Corollary 3.2(d) is the best possible in general is obtained by taking $F$ to be the field of real numbers, $K$ to be the field of complex numbers, and $I$ to be the ideal of $F[X]$ generated by $1-X_{1}^{2}-X_{2}^{2}-X_{3}^{2}$. In this case $1=\operatorname{pm~} S^{-1} B=\operatorname{dim} \max S^{-1} B / 2$.

In the remainder of this paper we are concerned with partial converses to Theorem 3.1.

TheOREM 3.3. Suppose $\left\{Q_{i}\right\}$ is a family of prime ideals of $B$ of maximal type. For each $i$, let $P_{i}=Q_{i} \cap A$. Let $S=B-\cup Q_{i}$ and $T=A-\cup P_{i}$. Suppose $T^{-1} B=S^{-1} B$. Then $\left\{P_{i}\right\}$ is a family of prime ideals of $A$ of maximal type.

Proof. It is sufficient to show that $\max T^{-1} A \subseteq\left\{P_{i}\left(T^{-1} A\right)\right\}$. Suppose $m$ is a maximal ideal of $T^{-1} A$. Since $T^{-1} B$ is an integral extension of $T^{-1} A$, there is a maximal ideal $Q_{i}\left(T^{-1} B\right)=Q_{i}\left(S^{-1} B\right)$ of $S^{-1} B$ which lies over $m$; that is, $m=\left(Q_{i} \cap A\right) T^{-1} A=P_{i}\left(T^{-1} A\right)$.

THEOREM 3.4. Suppose $B$, when regarded as an $A$-module, is finitely-generated and projective. Suppose $\left\{Q_{i}\right\}$ is a family of prime ideals of $B$ of maximal type such that there are no inclusions among the $Q_{i}$ 's and such that for each $i, Q_{i}$ is the unique prime ideal of $B$ which lies over the prime ideal $P_{i}=Q_{i} \cap A$ of $A$. Let $S=B-\cup Q_{i}$ and $T=A-\cup P_{i}$. Then:

(a) $S^{-1} B=T^{-1} B$.

(b) $\left\{P_{i}\right\}$ is a family of prime ideals of $A$ of maximal type such that there are no inclusion relations among the $P_{i}$ 's.

In the proof we need to recall some properties of the determinant, denoted det $f$, of an endomorphism $f$ of a finitely-generated projective $A$-module $M$ [2]. An $A$-module $N$ is chosen so that $M \oplus N=F$ is a finitely-generated free $A$ module. Let $g$ be the extension of $f$ to the endomorphism of $F$ which is the identity mapping on $N$. Then $\operatorname{det} f$ is defined to be det $g$. $f$ is an automorphism if and only if $\operatorname{det} f$ is a unit of $A$. If $T$ is a multiplicatively closed set of $A$ such that $0 \notin T$ and $1 \in T$, then the endomorphism $T^{-1} f$ of $T^{-1} P$ has the same determinant as $f$.

Proof. Let $s \in S$. Let $f: B \rightarrow B$ be the $A$-endomorphism defined by $f(b)=s b$ for each $b \in B$. It is sufficient to prove that $T^{-1} f$ is an automorphism; for if $g$ is the inverse of $T^{-1} f$, then $1=T^{-1} f(g(1))=s g(1)$ and then $s$ is a unit of $T^{-1} B$. Since det $T^{-1} f=\operatorname{det} f \in A$, it suffices to prove that det $f \notin P_{i}$ for all $i$. Fix $i$ and let $P=P_{i}$ and $Q=Q_{i}$. It follows from the hypothesis that $B_{P}$ has a unique maximal ideal $Q B_{P}$; hence $B_{P}=B_{Q}$. Since $s$ is a unit in $B_{Q},(B-Q)^{-1} f$ is an $A_{P}$-automorphism of $B_{Q}$ and $\operatorname{det} f$ $=\operatorname{det}(B-Q)^{-1} f$ is a unit in $A_{P}$. Hence det $f \notin P$. Condition (b) follows from Condition (a), Theorem 3.3 and the Cohen-Seidenberg going-up lemma.

\section{REFERENCES}

1. H. Bass, Algebraic K-theory, Benjamin, New York, 1968. MR 40 \#2736.

2. O. Goldman, Determinants in projective modules, Nagoya Math. J. 18 (1961), 27-36. MR 23 \# A 1671.

3. D. Lissner and K. Lønsted, Reduction of projective modulus in ring extensions (to appear). 
4. D. Lissner and N. Moore, Projective modules over certain rings of quotients of affine rings, J. Algebra 15 (1970), 72-80. MR 41 \# 1715.

5. N. Moore, Algebraic vector bundles over the 2-sphere, Invent. Math. 14 (1971), 167-172. MR 45 \#3403.

6. A. Simis, Projective moduli and maximal spectra of certain quotient rings, Trans. Amer. Math. Soc. 170 (1972), 125-136. MR 47 \#8513.

Department of Mathematics, Arizona State University, Tempe, Arizona 85281 